\title{
(2) OPEN ACCESS \\ Intracranial hematoma and abscess after neuraxial analgesia and anesthesia: a review of the literature describing 297 cases
}

\author{
Elke ME Bos $\odot{ }^{1},{ }^{1}$ Koen van der Lee, ${ }^{1}$ Johan Haumann, ${ }^{2}$ Marcel de Quelerij, ${ }^{3}$ \\ W Peter Vandertop (ㄷ, ${ }^{4}$ Cor J Kalkman, ${ }^{5}$ Markus W Hollmann (1) , ${ }^{1}$ Philipp Lirk ${ }^{6}$
}

\begin{abstract}
- Additional material is published online only. To view, please visit the journal online (http://dx.doi.org/10.1136/ rapm-2020-102154).
\end{abstract}

${ }^{1}$ Anesthesiology, Amsterdam UMC, Location AMC, Amsterdam, The Netherlands ${ }^{2}$ Anesthesiology, OLVG, Amsterdam, The Netherlands ${ }^{3}$ Anesthesiologie, Franciscus Gasthuis en Vlietland,

Rotterdam, The Netherlands ${ }^{4}$ Neurosurgical Center Amsterdam, Amsterdam UMC, Location AMC, Amsterdam, The Netherlands

${ }^{5}$ Anesthesiology, UMC Utrecht, Utrecht, The Netherlands ${ }^{6}$ Department of Anesthesiology, Brigham and Women's Hospital, Boston, Massachusetts, USA

\section{Correspondence to}

Professor Markus W Hollmann, Anesthesiology, Amsterdam UMC, Locatie AMC, Amsterdam 1100 DD, The Netherlands; m.w.hollmann@amc.uva.nl

Received 1 October 2020 Revised 21 December 2020 Accepted 22 December 2020 Published Online First 13 January 2021

\section{ABSTRACT \\ Background Besides spinal complications, intracranial hematoma or abscess may occur after neuraxial block. Risk factors and outcome remain unclear. \\ Objective This review evaluates characteristics, treatment and recovery of patients with intracranial complications after neuraxial block.}

Evidence review We systematically searched MEDLINE, Embase and the Cochrane Library from their inception to May 2020 for case reports/series, cohort studies and reviews of intracranial hematoma or abscess associated with neuraxial block. Quality of evidence was assessed using the critical appraisal of a case study checklist by Crombie.

Findings We analyzed 232 reports, including 291 patients with hematoma and six patients with abscess/ empyema. The major part of included studies comprised single case reports with a high risk of bias. Of the patients with hematoma, $48 \%$ concerned obstetric patients, the remainder received neuraxial block for various perioperative indications or pain management. Prior dural puncture was reported in $81 \%$, either intended (eg, spinal anesthesia) or unintended (eg, complicated epidural catheter placement). Headache was described in 217 patients; in 101 patients, symptoms resembled postdural puncture headache (PDPH). After treatment, $11 \%$ had partial or no recovery and $8 \%$ died, indicating the severity of this complication. Intracranial abscess after neuraxial block is seldom reported; six reports were found.

Conclusion Diagnosis of intracranial hematoma is often missed initially, as headache is assumed to be caused by cerebrospinal hypotension due to cerebrospinal fluid leakage, known as PDPH. Prolonged headache without improvement, worsening symptoms despite treatment or epidural blood patch, change of headache from postural to non-postural or new neurological signs should alert physicians to alternative diagnoses.

\section{INTRODUCTION}

Neuraxial administration of local anesthetics is widely used to provide analgesia or anesthesia. The most frequently reported side effects of neuraxial block, such as periprocedural hypotension, urine retention and postdural puncture headache (PDPH), are usually self-limiting or relatively easy to treat. More serious complications, such as spinal hematoma or abscess, although rare, feature prominently in the anesthesia literature as they may result in permanent neurological injury. ${ }^{1-5}$ Intracranial complications, such as hematoma or abscess, may not be instantly recognized as complications of neuraxial analgesia, even though the consequences can be significant as well, with a possibility of permanent neurological deficit despite treatment.

Literature reviews focusing on the development of intracranial hematomas following neuraxial anesthesia are scarce and have mainly focused on obstetric patients as the incidence seems highest in this population. ${ }^{6-9}$ The few reviews that are present in other populations, for example, perioperative patients, are small and non-systematic. ${ }^{10} 11$

Previously, we systematically reviewed spinal complications after neuraxial block. ${ }^{5}$ Using a similar approach, we aimed to collect all cases reported in the literature concerning intracranial hematomas or abscesses following neuraxial block, in order to identify possible predisposing patient characteristics, to describe the ensuing clinical course and to gain insight under which circumstances complications would be most likely to occur.

\section{METHODS}

\section{Search strategy}

A systematic search in MEDLINE, Embase and the Cochrane Central Register of Controlled Trials from inception of databases to May 11, 2020, was performed to identify relevant studies. The search consisted of controlled vocabulary (ie, Medical Subject Headings in MEDLINE) and free-text words for neuraxial blocks and intracranial hematomas and abscesses. Animal studies were safely excluded by double negation (not exp animals/not humans/). No further language, date or other restrictions were imposed (for the entire MEDLINE search strategy, see online supplemental file 1). We cross-checked the reference lists and the citing articles of the identified relevant papers for additional references. The bibliographical records retrieved were imported and de-duplicated in EndNote V.X9.3.3 (Clarivate Analytics, USA).

\section{Article selection}

Titles, abstracts and subsequently full texts were independently screened for reports concerning intracranial hematomas and abscesses associated with neuraxial block by two authors (EMEB and $\mathrm{KvdL}$ ). Inclusion criteria for eligibility were intracranial hematoma or abscess after neuraxial block in humans. We defined intracranial hematoma or 


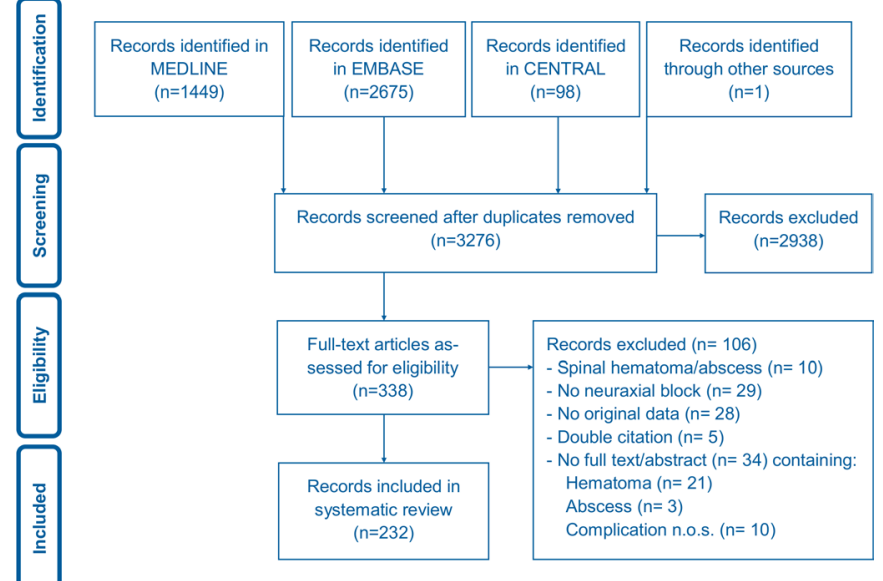

Figure 1 Flowchart of search and selection of records concerning intracranial hematomas and abscesses after neuraxial block from inception of databases until 11 May 2020. n, number of articles, n.o.s., not otherwise specified.

abscess as any epidural, subdural, subarachnoid or intraparenchymal hematoma or abscess above the level of C0. Neuraxial blocks were classified as continuous epidural analgesia, spinal anesthesia, a combined spinal-epidural (CSE), epidural injection, spinal catheters and spinal cord stimulators. Case reports, case series, prospective and retrospective cohort studies, systematic reviews and literature reviews (if containing original data) in English, Dutch, French or German were included. When articles in other languages were encountered but an English abstract was found, we restricted data extraction to the abstract. We confirmed that no overlap was present between cases described in reviews or cohort studies and case reports by screening data of patients with, for instance, identical age, comorbidities, author names and clinical affiliations. When all variables were identical and the presence of a double citation was confirmed, one of the identical citations was excluded (see figure 1, double citation). All cases with neuraxial block prior to the development of intracranial hematoma or abscess were included; also cases where causality of the complication to neuraxial block was uncertain and may be explained by underlying disease (ie, vascular malformations as aneurysms and arteriovenous malformations) were included.

\section{Quality assessment}

We used the critical appraisal of a case study checklist, adapted from The Pocket Guide to Critical Appraisal by Crombie, to assess the quality of the included studies. ${ }^{12}$ Two authors (EMEB and $\mathrm{KvdL}$ ) independently assessed the quality of all publications reporting more than one case. The quality of single case reports was not assessed because of likely selection and publication bias.

\section{Data extraction}

Two independent reviewers (EMEB and KvdL) extracted information from the selected articles. When available, extracted data included space of complication (epidural, subdural, subarachnoid or intraparenchymal), location of complication (unilateral/ bilateral; unilateral hematoma/abscess describes a complication located in one hemisphere, while bilateral hematomas/abscesses describe complications located in both hemispheres of the brain), age, sex, Body Mass Index, American Society of Anesthesiologists (ASA) physical status, ${ }^{13}$ coagulation status, indication for neuraxial block, type of neuraxial procedure, needle size, report of complicated puncture, presenting symptoms, timeline from onset of neuraxial block to complication, moment of first symptoms (rounded to full hours), moment of first neurological symptoms (rounded to full hours), type of treatment, timing of evacuation of hematoma or abscess if performed (rounded to full hours) and recovery after treatment (for complete information on extracted data, see online supplemental file 2).

Clinical symptoms prior to treatment decision were scored, based on symptoms described in the reported cases. Globally, all patients were scored for the presence of pain (ie, headache/neck pain), seizures, nausea/vomiting/dizziness, aphasia/dysarthria, visual disturbance, drowsiness/disorientation and neurological deficits (motor and sensory disturbances). We classified the extent of neurological deficit further using the Glasgow Coma Scale ${ }^{14}$ (GCS); a GCS score of $<13$ was considered to be disturbed. Neurological symptoms, as indicated in the variable 'moment of first neurological symptoms', comprised motor, sensory or GCS disturbances. When first presenting symptoms comprised neurological deficits, the variables 'moment of first symptoms' and moment of first neurological symptoms were scored identical, when first presenting symptoms comprised symptoms other than motor/sensory/GCS disturbances (eg, headache), the moment of first symptoms was scored different from the moment of first neurological symptoms (if any).

Regarding type of treatment, a distinction was made between neurosurgical decompression and conservative management. Neurosurgical decompression consisted of burr holes, craniotomy or other neurosurgical decompressive intervention. Conservative management was described to comprise treatment with analgesics, corticosteroids (eg, dexamethasone), antibiotics, physiotherapy, rehabilitation or no treatment at all.

Recovery after treatment was scored as 'full recovery', 'delayed but full recovery' (full recovery after more than one month from symptom onset), 'partial recovery' (improvement of symptoms, but persistent neurological deficit or pain present after treatment) and 'no objective recovery after treatment'.

\section{Statistical analysis}

SPSS V.26.0 was used for statistical analyses. Normally distributed continuous variables are presented as mean (SD) and were compared using independent samples t-tests, whereas nonnormally distributed variables are presented as medians (IQR) and compared using Mann-Whitney tests. Categorical variables are presented as n (\%) and were compared using Pearson $\chi^{2}$ tests, Fisher exact test or Fisher-Freeman-Halton exact test.

A two-sided $\mathrm{p}$ value of 0.05 was considered statistically significant.

\section{RESULTS}

The search retrieved 3276 publications, from which we selected 338 records for assessment of eligibility. Finally, we included 232 records in this review. Intracranial hematoma after neuraxial block was reported in 291 patients (226 articles); 9 patients suffered from a combination of spinal and intracranial hematoma. ${ }^{15-23}$ Intracranial abscess was reported in six patients (six articles); one patient suffered from both spinal and intracranial abscesses. ${ }^{24}$ Data collection and selection of articles are described in figure 1. Articles concerning patients with intracranial hematoma included 14 case series/cohort studies reporting 79 patients and 212 patients in single case reports. Patients with abscess/empyema were reported in five single case reports and in one closed claims analysis also describing other serious 
complications after neuraxial anesthesia, including spinal hematoma and abscess.

\section{Quality assessment}

A large proportion of included studies comprised single case reports; therefore, the risk of bias in the included studies was high. Furthermore, the quality of the case series and cohort studies was questionable, as many variables of the checklist ${ }^{12}$ were scored 'unclear' or 'negative'. Information on the quality of each of the case series and cohort studies is shown in online supplemental file 3 . Among the 14 studies (13 articles reporting solely patients with intracranial hematoma and 1 article reporting intracranial abscess but also reporting spinal hematoma and abscess, which are not included in this analysis), only 1 study scored positive on 9 of 11 required items of the checklist. None of the case series or cohort studies scored positive on all items of the checklist.

\section{Intracranial hematoma}

Characteristics of patients with intracranial hematoma are described in table 1 . Patients were generally young and healthy, with a median age of $34(28-50)$ years and an ASA physical status of 2 in 119 of 146 cases with data on ASA physical status; data were missing in 145 cases (50\%). Approximately half of patients comprise obstetric patients either undergoing cesarian section or receiving neuraxial labor analgesia (141 of 291 patients, 48\%).

Most intracranial hematomas occurred after intended dural punctures, that is, spinal anesthesia, spinal catheter or CSE procedures (if intending spinal anesthesia). Unintended dural puncture (eg, spinal tap when attempting to place an epidural catheter for epidural analgesia or CSE) occurred in 51 patients; in 32 of 77 patients (42\%) treated with epidural analgesia, in 7 patients who experienced a failed regional technique, in 3 patients during epidural injection, in 1 patient treated with a CSE procedure and in 1 patient who received a spinal cord stimulator; furthermore, it was decided to place a spinal catheter after unintended dural puncture while attempting different neuraxial blocks in an additional 7 patients (epidural analgesia was intended in 5 of these patients and CSE in 2 patients). In this series, 237 of 291 patients (81\%) experienced a dural puncture, either intended or unintended.

No interference with the coagulation system (ie, medication or underlying disease) was noticed in 74 patients, 25 patients used prophylactic low-molecular-weight heparin, aspirin/clopidogrel, direct oral anticoagulants, a combination of anticoagulants, had heparin infusion or a coagulation disorder, or anticoagulant therapy was unspecified (see table 1); interference with the coagulation system was unreported in 192 cases (66\%).

\section{Clinical presentation}

Virtually all patients reported pain (217 of 220 patients with information on the presence or absence of pain), that is, headache and/or neck pain. In 101 of 217 patients with pain, the headache complied with criteria for (or was initially confused with) $\mathrm{PDPH}^{25}$; in 16 patients, the headache did not adhere to the criteria for PDPH and an alternative diagnosis was considered, and in the remaining 100 of 217 patients with pain, detailed information was missing. Sensory disturbances or motor deficits were present in $17 \%$ and $27 \%$, respectively. Sensory and motor symptoms were diverse and could comprise mild neurological symptoms, that is, numbness or mild muscle weakness in the face or extremities ${ }^{17} 26-30$ or severe neurological deficit such as hemiparesis, ${ }^{31}$ tetraparesis due to
Table 1 Characteristics of patients with intracranial hematoma associated with neuraxial block

Intracranial hematoma: patient

\begin{tabular}{|c|c|c|}
\hline characteristics & Total: 291 & $\mathbf{n}$ \\
\hline Age (years), median (IQR) & $34.0(28.0-50.0)$ & 240 \\
\hline Sex (male, female, missing), $\mathrm{n}(\%)$ & 60 (20.6), $187(64.3), 44(15.1)$ & 247 \\
\hline BMI $\left(\mathrm{kg} / \mathrm{m}^{2}\right)$, median (IQR) & $25.5(23.5-29.8)$ & 30 \\
\hline ASA physical status, $\mathrm{n}(\%)$ & & 146 \\
\hline Class 1 & $13(4.5)$ & \\
\hline Class 2 & $119(40.9)$ & \\
\hline Class 3 & $12(4.1)$ & \\
\hline Class 4 & $2(0.7)$ & \\
\hline Missing & $145(49.8)$ & \\
\hline Neuraxial technique & & 290 \\
\hline Spinal anesthesia & $123(42.3)$ & \\
\hline Epidural catheter & $77(26.5)$ & \\
\hline Spinal catheter & $57(19.6)$ & \\
\hline Combined spinal-epidural procedure & $14(4.8)$ & \\
\hline Failed regional technique & $12(4.1)$ & \\
\hline Epidural injection & $6(2.1)$ & \\
\hline Spinal cord stimulator & $1(0.3)$ & \\
\hline Missing & $1(0.3)$ & \\
\hline Needle size (gage) & & 118 \\
\hline$<18$ & $20(6.9)$ & \\
\hline $18-21$ & $25(8.6)$ & \\
\hline $22-24$ & $23(7.9)$ & \\
\hline $25-27$ & $50(17.2)$ & \\
\hline Missing & $173(59.5)$ & \\
\hline
\end{tabular}

Report of complicated puncture

No difficult puncture

$71(24.4)$

Difficult/multiple punctures $27(9.3)$

Bloody tap $1(0.3)$

Unintended dural puncture 51 (17.5)

Missing 141 (48.5)

Procedure

Obstetric: cesarian section $\quad 76(26.1)$

Obstetric: labor analgesia $\quad 65$ (22.3)

Aneurysm repair (open or endovascular) 42 (14.4)

Minor digestive 24 (8.2)

Pain management $\quad 14(4.8)$

Urological $13(4.5)$

Major orthopedic $12(4.1)$

Gynecological oncology/surgery $8(2.7)$

Minor orthopedic $6(2.1)$

Peripheral vascular $\quad 4(1.4)$

Major digestive $3(1.0)$

Thoracic (thoracotomy/thoracoscopy) $2(0.7)$

Amputation (leg)/extremities $\quad 1(0.3)$

Missing $21(7.2)$

Coagulation status

99

No regular anticoagulants $\quad 74(25.4)$

Prophylactic LMWH $10(3.4)$

Aspirin/clopidogrel $6(2.1)$

Coagulation disorder $4(1.4)$

Heparin infusion $2(0.7)$

DOAC $1(0.3)$

Unspecified anticoagulant therapy $\quad 1(0.3)$

LMWH and vitamin $\mathrm{K}$ antagonist $\quad 1(0.3)$

Missing $192(66.0)$

Time point of complication* 


\begin{tabular}{|c|c|c|}
\hline $\begin{array}{l}\text { Intracranial hematoma: patient } \\
\text { characteristics }\end{array}$ & Total: 291 & $\mathrm{n}$ \\
\hline During puncture & $27(9.3)$ & \\
\hline During treatment & $19(6.5)$ & \\
\hline After removal of catheter & $58(19.9)$ & \\
\hline After spinal anesthesia & $124(42.6)$ & \\
\hline Missing & $63(21.6)$ & \\
\hline Space & & 237 \\
\hline Subdural & $200(68.7)$ & \\
\hline Subarachnoidal & $18(6.2)$ & \\
\hline Intraparenchymal & $12(4.1)$ & \\
\hline Epidural & $3(1.0)$ & \\
\hline Subdural and subarachnoidal & $4(1.4)$ & \\
\hline Missing & $54(18.6)$ & \\
\hline Unilateral, bilateral, missing, $\mathrm{n}(\%)$ & $119(40.9), 46(15.8), 126(43.3)$ & 165 \\
\hline \multicolumn{3}{|l|}{ Symptoms } \\
\hline $\begin{array}{l}\text { Pain (headache/neck pain) (present, } \\
\text { absent, missing) }\end{array}$ & 217 (74.6), $3(1.0), 71$ (24.4) & 220 \\
\hline $\begin{array}{l}\text { Sensory deficit (present, absent, } \\
\text { missing) }\end{array}$ & 50 (17.2), $128(44.0), 113(38.8)$ & 178 \\
\hline Motor deficit (present, absent, missing) & 79 (27.1), $131(45.0), 81(27.8)$ & 210 \\
\hline PDPH (present, absent, missing) & 101 (34.7), 16 (5.5), 174 (59.8) & 117 \\
\hline Seizures (present, absent, missing) & $27(9.3), 27(9.3), 237$ (81.4) & 54 \\
\hline $\begin{array}{l}\text { Aphasia/dysarthria (present, absent, } \\
\text { missing) }\end{array}$ & 19 (6.5), 26 (8.9), 246 (84.5) & 45 \\
\hline $\begin{array}{l}\text { Visual disturbance (present, absent, } \\
\text { missing) }\end{array}$ & 37 (12.7), 23 (7.9), 231 (79.4) & 60 \\
\hline $\begin{array}{l}\text { Vomiting/nausea/dizziness (present, } \\
\text { absent, missing) }\end{array}$ & 74 (25.4), 15 (5.2), 202 (69.4) & 89 \\
\hline $\begin{array}{l}\text { Drowsy/disorientation (present, absent, } \\
\text { missing) }\end{array}$ & $49(16.8), 24(8.2), 218(74.9)$ & 73 \\
\hline $\begin{array}{l}\text { GCS disturbed (eg, <13) (present, } \\
\text { absent, missing) }\end{array}$ & 60 (20.6), 33 (11.3), 198 (68.0) & 93 \\
\hline $\begin{array}{l}\text { GCS count if GCS disturbed, median } \\
\text { (IQR) }\end{array}$ & $5.0(3.0-7.0)$ & 24 \\
\hline Treatment, n (\%) & & 256 \\
\hline Conservative & $144(49.5)$ & \\
\hline Neurosurgical decompression & $112(38.5)$ & \\
\hline Missing & $35(12.0)$ & \\
\hline EBP (yes, no, missing), n (\%) & $57(19.6), 24$ (8.2), $210(72.2)$ & 81 \\
\hline Recovery after treatment, $\mathrm{n}(\%)$ & & 216 \\
\hline Full recovery & $117(40.2)$ & \\
\hline Delayed but full recovery & $45(15.5)$ & \\
\hline Partial recovery & $19(6.5)$ & \\
\hline No recovery & $13(4.5)$ & \\
\hline Death & $22(7.6)$ & \\
\hline Missing & $75(25.8)$ & \\
\hline
\end{tabular}

*'During puncture': symptoms during/directly after puncture, including epidural analgesia, SCS, continuous spinal anesthesia (spinal catheter) and failed regional techniques; 'during drug administration': when drugs were administered through a catheter or treatment with SCS was ongoing; 'after removal of catheter': if catheter or SCS was removed.

ASA, American Society of Anesthesiologists; BMI, Body Mass Index; DOAC, direct ora anticoagulants; EBP, epidural blood patch; GCS, Glasgow Coma Scale; LMWH, lowmolecular-weight heparin; $n$, cases with available data on specific parameter; PDPH, postdural puncture headache; SCS, spinal cord stimulator.

bilateral parietal subdural hematomas ${ }^{32}$ or a comatose state. ${ }^{33}$ The GCS score was abnormal in 60 patients.

Concerning the 16 patients for which the clinician considered diagnoses different from PDPH, symptoms that were considered to be less suggestive of PDPH were the presence of a constant headache, position independent headache, headache that intensified in lying position or diminished in sitting position, or headache occurring during/immediately after puncture or after more than 14 days after neuraxial block. GCS was disturbed in five of these 16 patients, motor disturbances and sensory disturbances were reported in seven and five patients, respectively. Seven patients reported to have nausea/vomiting/dizziness, in six patients drowsiness/disorientation was described, three patients were described to have seizures, three patients had visual disturbances, and two of these patients presented with aphasia/ dysarthria.

\section{Development of symptoms (timeline)}

The median time to symptom onset after neuraxial block (ie, all symptoms, for instance, pain, nausea/vomiting or neurological deficits such as motor/sensory/GCS disturbances) was 36 hours (10-72, $n=204)$; the first neurological symptoms (comprising motor/sensory/GCS disturbances) occurred after 120 hours $(48-276, n=112)$, the median time of symptom progression was 120 hours $(24-427, n=160)$. The time between start of first symptoms (often headache) and evacuation of hematoma, if performed, was 197 hours $(7-518, n=85)$; the time between development of neurological symptoms to evacuation was shorter, 4 hours $(3-24, n=63)$; and the time between diagnosis and evacuation, if performed, was 2 hours $(1-4, n=79)$.

\section{Treatment and recovery}

After intracranial hematoma was diagnosed, 112 patients were treated with neurosurgical drainage and 144 patients were treated conservatively; this information was missing in the remaining 35 patients. Overall, the majority of patients had full recovery (162 of 291 patients, 56\%); 32 patients (11\%) had permanent sequelae after treatment; 22 patients died (8\%); and information regarding recovery was missing in 75 patients $(26 \%)$.

Patients with permanent sequelae after treatment remained with a variability of symptoms, ranging from ptosis, epilepsy, persistent paralysis or a remaining comatose state after symptom progression. Regarding the 22 patients who died due to the complication, many of these patients presented initially with or without mild neurological symptoms; however, after a period of vague complaints, a sudden and rapid progression to unconsciousness occurred, finally leading to brain herniation and death (median hours of symptom progression of 72 hours, 13-236; $\mathrm{n}=17$ ). Patients who died were generally older than survivors, with a median age of $66(38-70)$ years $(n=21)$ vs $34(28-42)$ years $(\mathrm{n}=186)$, respectively $(\mathrm{p}<0.001)$, and were more often male $(52 \%, \mathrm{n}=21$, vs $20 \%, \mathrm{n}=191$, respectively; $\mathrm{p}<0.01)$.

Fifty-five of 101 patients diagnosed with PDPH were treated with an epidural blood patch (EBP) to alleviate the headache; 10 patients were treated without EBP, and this information was missing in the remaining patients. One patient without PDPH symptoms but with confirmed bilateral subdural hematoma was treated with an EBP to stop ongoing cerebrospinal fluid (CSF) leakage, ${ }^{34}$ and one patient who was already treated twice with craniotomy for recurrent intracranial hematoma received an EBP because of ongoing CSF leakage from a lumbar drain site ${ }^{35}$; both cases resulted in complete recovery. In the limited number of patients with reported data regarding the performance of an EBP and outcome, no differences were seen in terms of recovery $(\mathrm{n}=73, \mathrm{p}=0.13)$.

\section{Intracranial abscess/empyema}

The characteristics of six patients with intracranial abscess after neuraxial anesthesia previously reported in the literature are 


\begin{tabular}{|c|c|c|}
\hline Intracranial abscess: patient characteristics & Total: 6 & $\mathbf{n}$ \\
\hline Age (years), median (IQR) & $32.0(24.3-65.3)$ & 6 \\
\hline Sex (male, female), n (\%) & 1 (16.7), 5 (83.3) & 6 \\
\hline ASA physical status, $n(\%)$ & & 4 \\
\hline Class 1 & $0(0.0)$ & \\
\hline Class 2 & $3(50.0)$ & \\
\hline Class 3 & $0(0.0)$ & \\
\hline Class 4 & $1(16.7)$ & \\
\hline Missing & $2(33.3)$ & \\
\hline Neuraxial technique & & 6 \\
\hline Spinal anesthesia & $4(66.7)$ & \\
\hline Epidural catheter & $1(16.7)$ & \\
\hline Spinal catheter & $1(16.7)$ & \\
\hline Report of complicated puncture & & 2 \\
\hline No difficult puncture & $2(33.3)$ & \\
\hline Missing & $4(66.7)$ & \\
\hline Procedure & & 6 \\
\hline Obstetric: cesarian section & $4(66.7)$ & \\
\hline Pain management & $1(16.7)$ & \\
\hline Minor orthopedic & $1(16.7)$ & \\
\hline Time point of complication* & & 6 \\
\hline During puncture & $0(0.0)$ & \\
\hline During treatment & $1(16.7)$ & \\
\hline After removal of catheter & $1(16.7)$ & \\
\hline After spinal anesthesia & $4(66.7)$ & \\
\hline \multicolumn{3}{|l|}{ Symptoms } \\
\hline Pain (headache/neck pain) (present, absent, missing) & $5(83.3), 1(16.7), 0(0.0)$ & 6 \\
\hline PDPH (present, absent, missing) & $2(33.3), 1(16.7), 3(50.0)$ & 3 \\
\hline Seizures (present, absent, missing) & $1(16.7), 2(33.3), 3(50.0)$ & 3 \\
\hline Aphasia/dysarthria (present, absent, missing) & $1(16.7), 1(16.7), 4(66.7)$ & 2 \\
\hline Visual disturbance (present, absent, missing) & $1(16.7), 2(33.3), 3(50.0)$ & 3 \\
\hline Vomiting/nausea/dizziness (present, absent, missing) & $0(0.0), 2(33.3), 4(66.7)$ & 2 \\
\hline Drowsy/disorientation (present, absent, missing) & $1(16.7), 1(16.7), 4(66.7)$ & 2 \\
\hline Fever (present, absent, missing) & $5(83.3), 0(0.0), 1(16.7)$ & 5 \\
\hline Treatment, n (\%) & & 6 \\
\hline Conservative & $2(33.3)$ & \\
\hline Neurosurgical decompression & $4(66.7)$ & \\
\hline Recovery after treatment, $\mathrm{n}(\%)$ & & 6 \\
\hline Full recovery & $4(66.7)$ & \\
\hline Delayed but full recovery & $0(0.0)$ & \\
\hline Partial recovery & $0(0.0)$ & \\
\hline No recovery & $0(0.0)$ & \\
\hline Death & $2(33.3)$ & \\
\hline
\end{tabular}

*'During puncture': symptoms during/directly after puncture, including epidural analgesia, SCS, continuous spinal anesthesia (spinal catheter) and failed regional techniques; 'during drug administration': when drugs were administered through a catheter or treatment with SCS was ongoing; 'after removal of catheter': if catheter or SCS was removed. ASA, American Society of Anesthesiologists; $n$, cases with available data on specific parameter; PDPH, postdural puncture headache; SCS, spinal cord stimulator.

described in table 2 . Most patients were relatively young, with a median age of 32 (24-65) years; five were female, and the indication for neuraxial block was a cesarian section in four cases. Most patients reported headache and/or neck pain (five patients). The median time to symptom onset after neuraxial block was 228 hours $(48-4170, n=6)$. Four cases were treated with neurosurgical decompression of abscess(es) combined with antibiotic treatment and two cases were treated conservatively, including antibiotic treatment. Cultures (blood cultures or culture of the surgical specimen) resulted in Mycoplasma hominis in two cases, Streptococcus anginosus in one case and Aspergillus in one case, and cultures remained negative in the two remaining cases. Overall, four patients recovered fully, while two patients died from the complication.

In one of the reported cases, a brain abscess developed 17 months after implantation of an intrathecal drug delivery device for severe cancer-related rectal and perineal pain in a man in his mid-70s with metastatic anal cancer, after abdominoperineal resection, chemotherapy and radiation therapy. ${ }^{36}$ The implanted device might have been contaminated and thereby might have contributed to the development of the abscess; however, a possible other cause included hematogenous spread of infection in a patient with chemotherapy-induced low immune response, indicating an uncertain causality between the neuraxial procedure and the development of a brain abscess.

\section{DISCUSSION}

We analyzed the literature of intracranial hematomas and abscesses after neuraxial block and identified 291 cases of hematoma and 6 cases of abscess or empyema.

Intracranial hematomas were reported predominantly after puncture of the dura mater in relatively young and healthy women receiving neuraxial block for obstetric indications. Besides obstetric patients, intracranial hematoma was reported in diverse patient populations, including patients treated with a spinal catheter for aneurysm repair or with neuraxial block for surgical indications or pain management.

Virtually every patient with an intracranial hematoma reported headache. In the majority of patients in this series, a dural puncture had taken place, either intended (eg, spinal anesthesia) or unintended (eg, spinal tap when attempting to place an epidural catheter). Within this review, many patients reported symptoms compatible with PDPH. Typical characteristics of PDPH, described by the International Classification of Headache disorders, ${ }^{25}$ are headache occurring within 5 days of a lumbar puncture, remitting spontaneously within 2 weeks or after sealing of the leak with an autologous epidural lumbar patch, and usually (but not invariably) the headache is orthostatic. Studies that evaluated the time course of PDPH demonstrated that in approximately $72 \%-95 \%$ of cases, the symptoms subsided within 5-7 days. ${ }^{1137}$ Our analysis of the literature showed a median time lapse between neuraxial block and first neurological symptoms of 5 days, indicating that a diagnosis of PDPH is unlikely at that point in time or that ongoing CSF leakage may have led to secondary complications, such as intracranial bleeding. Thus, when PDPH is prolonged for more than 5 days (and especially for more than 2 weeks), does not improve or worsens with clinical treatment or after an EBP, if changes in pain occur from postural to non-postural, or if neurological signs or symptoms develop besides the headache, the occurrence of other causes of headache, for example, intracranial hematoma, should be considered. ${ }^{1129}$ Neurological consultation and imaging studies are indicated at this point in the clinical course of falsely presumed PDPH, ideally before neurological symptoms occur, to prevent progression of underlying disease and delayed treatment. These findings are in line with previous studies assessing characteristics of patients with intracranial hematoma and PDPH after neuraxial procedures in the obstetric population. ${ }^{633}$ Cuypers et al, accentuate that patients who develop a persistent headache after neuraxial procedures require careful follow-up, even in the absence of predisposing risk factors or an obvious dural puncture. ${ }^{6}$ Also Lim et al emphasize the need for close monitoring of patients who have PDPH after neuraxial anesthesia for signs that could signal the evolution of intracranial hematoma, based on 
their case series of 11 patients with subdural hematomas associated with the use of labor epidural analgesia over 7 years at a tertiary care hospital. ${ }^{33}$ Interestingly, in their series, most cases of subdural hematoma did not manifest with significant additional neurological changes beyond typical clinical symptoms of PDPH (10 of 11 patients), which differs from our results describing sensory disturbances or motor deficit in $17 \%$ and $27 \%$, respectively, and a disturbed GCS in $21 \%$ of patients. A possible explanation for the higher number of patients presenting with neurological deficits in our literature review could be reporting bias; it may be more interesting to report severe complications as opposed to patients with mild symptoms and positive outcome. In general, PDPH may be associated with substantially increased risks of major neurological complications and other maternal complications in obstetric patients, underscoring the need for early recognition, treatment and follow-up of patients with PDPH. ${ }^{9}$ Intracranial abscess after neuraxial block seems to be very seldom and is hardly ever reported in literature. It is impossible to yield any conclusion based on the limited data.

\section{Pathophysiology}

The pathophysiology of intracranial hematoma is based on craniospinal hypotension due to CSF leakage from the dural puncture site, which - if severe - may lead to a caudal shift of the brain, causing bridging veins to tear. ${ }^{6}$ As discussed earlier, ongoing CSF leakage without sealing of the leak with an autologous epidural lumbar blood patch may lead to intracranial hematoma. Furthermore, another possibility is that intracranial bleeding occurred regardless of CSF leakage, or the diagnosis PDPH was falsely presumed and intracranial hematoma was present in the first place. The use of an EBP for suspected ongoing CSF leakage can be effective, however, in the presence of confirmed intracranial hematoma, this may lead to increased intracranial pressure with further deterioration as a result. Epidural blood patching in the presence of confirmed cranial hematomas should therefore be used cautiously and only after consultation with a neurologist or neurosurgeon.

Of note, the incidence of unrecognized dural puncture at the time of epidural procedures is considerable, with reported rates of occurrence between $10 \%$ and $36 \% .{ }^{3839}$ CSF leakage and subsequent PDPH or intracranial hematoma can occur after puncture with larger needles used for placement of epidural or spinal catheters, but also after puncture with smaller/thin needles used for spinal anesthesia. Other factors that may increase bleeding risk are pre-existing cranial vascular anomalies such as aneurysms or arterial venous malformations, ${ }^{1640}$ cerebral venous thrombosis, hypertension, brain tumors, coagulation disorders, hematological disorders, or anticoagulant and thrombolytic therapy. ${ }^{41}$

In general, intracranial hematoma seems to be more common in pregnancy and during the direct postpartum period than in nonpregnant women of comparable age. ${ }^{42} 43$ This may be related to an increased circulating volume, presence of pre-existing cranial vascular anomalies, coagulopathy or hypertensive disorders such as preexisting hypertension and pre-eclampsia/eclampsia. ${ }^{42-44}$ The effect of a neuraxial block on the risk of intracranial bleeding in pregnant or direct postpartum women is unknown.

The pathophysiology of intracranial abscesses or empyema after neuraxial block seems to originate from meningitis, which progresses to (mostly subdural) empyema or brain abscesses, or an infected hematoma that develops into an abscess. The infection can occur at the time of neuraxial procedure, or through contamination of the skin site and subsequent spread along a spinal or epidural catheter (by skin flora of the patient or in exceptional cases skin flora of a treating physician), by hematological spread or by intraluminal contamination via a polluted syringe or local anesthetic solution. ${ }^{45}$

Overall, a high level of suspicion for the potential relationship between symptoms indicative of a complication and the previous neuraxial block should be present, as first symptoms may resemble alternative diagnoses (such as typical PDPH) and occur when patients are no longer under the care of an anesthesiologist or an acute pain service physician. ${ }^{5}$

\section{Limitations}

The retrospective character and selective reporting of case reports and case series resulted in multiple limitations. For instance, lack of detail in the reports was a major limitation, the quality of the included studies was low, and, as in accordance with retrospective research, a high risk of publication bias, selection bias and under-reporting of complications is present. While acknowledging these limitations, we feel that the present analyses of aggregated reported cases can improve our understanding of the development of these rare but severe complications of neuraxial block.

Specifically, we noted that many reports described widely diverse aspects of the clinical course, treatment and outcome of intracranial hematoma or abscess leading to large quantities of missing data. This is in congruence with the findings of our previous literature review analyzing spinal hematoma and abscess after neuraxial block. ${ }^{5}$ No consensus appears to exist on the most important aspects of these severe complications. Again we want to suggest that registration of complications after neuraxial block and the reporting of cases should be compliant with a predefined format (see online supplemental file 2), to collect complete data allowing more accurate estimates of incidence rates, prognostic factors and response to therapy. ${ }^{5}$

\section{CONCLUSION}

Intracranial hematoma is a rare but possible complication after neuraxial block and mainly occurs after puncture of the dura mater. The diagnosis intracranial hematoma is often missed initially, as the headache is presumed to be caused by the much more common cerebrospinal hypotension syndrome after dural CSF leakage. When the headache is prolonged for more than 5 days, does not improve, or worsens, with clinical treatment or after EBP, changes from postural to non-postural, or if neurological symptoms develop besides the headache, alternative diagnoses should be considered warranting neurological consultation and imaging studies.

Acknowledgements We thank J Limpens, medical librarian (Amsterdam UMC, location Academic Medical Center, Amsterdam), for the comprehensive literature search, and S van Dieren, clinical epidemiologist (Amsterdam UMC, location Academic Medical Center, Amsterdam), for the support in the statistical analysis.

Contributors EMEB, KvdL and JH: literature search and data collection. EMEB, KvdL, JH and PL: data analysis. EMEB: drafting of the article. All authors: study concept and design; interpretation of data; revision of the draft critically for important intellectual content; final approval of the version to be published; and agreement to be accountable for all aspects of the work, thereby ensuring that questions related to the accuracy or integrity of any part of the work are appropriately investigated and resolved.

Funding This study was supported by departmental funding

Competing interests MdQ: received payments for lectures from Johnson \& Johnson Medical Devices Companies. MWH: executive section editor, Pharmacology with Anesthesia \& Analgesia, and section editor, Anesthesiology, with Journal of Clinical Medicine; furthermore, he served as consultant for Euro-cept BV and received honoraria for lectures from CSL Behring in the past.

Patient consent for publication Not required. 
Provenance and peer review Not commissioned; externally peer reviewed.

Data availability statement Data are available upon reasonable request. Data comprise patient data as reported in previously published case reports, case series, cohort studies and reviews (if containing original data).

Open access This is an open access article distributed in accordance with the Creative Commons Attribution Non Commercial (CC BY-NC 4.0) license, which permits others to distribute, remix, adapt, build upon this work non-commercially, and license their derivative works on different terms, provided the original work is properly cited, an indication of whether changes were made, and the use is noncommercial. See: http://creativecommons.org/licenses/by-nc/4.0/.

\section{ORCID iDs}

Elke ME Bos http://orcid.org/0000-0003-3743-3435

W Peter Vandertop http://orcid.org/0000-0001-5417-0265

Markus W Hollmann http://orcid.org/0000-0001-8248-0244

\section{REFERENCES}

1 Su J, Soliz JM, Popat KU, et al. Complications of postoperative epidural analgesia for oncologic surgery: a review of 18,895 cases. Clin J Pain 2019;35:589-93.

2 Rosero EB, Joshi GP. Nationwide incidence of serious complications of epidural analgesia in the United States. Acta Anaesthesio/ Scand 2016;60:810-20.

3 Christie IW, McCabe S. Major complications of epidural analgesia after surgery: results of a six-year survey. Anaesthesia 2007;62:335-41.

4 Brull R, McCartney CJL, Chan VWS, et al. Neurological complications after regional anesthesia: contemporary estimates of risk. Anesth Analg 2007;104:965-74.

5 Bos EME, Haumann J, de Quelerij M, et al. Haematoma and abscess after neuraxial anaesthesia: a review of 647 cases. Br J Anaesth 2018;120:693-704.

6 Cuypers V, Van de Velde M, Devroe S. Intracranial subdural haematoma following neuraxial anaesthesia in the obstetric population: a literature review with analysis of 56 reported cases. Int J Obstet Anesth 2016;25:58-65.

7 Turk CC, Uyar R, Kara NN. Characteristics of intracranial subdural hematomas following spinal and epidural anesthesia in obstetric patients. J Neurol Sci 2016;33:341-51.

8 Moore AR, Wieczorek PM, Carvalho JCA. Association between Post-Dural puncture headache after neuraxial anesthesia in childbirth and intracranial subdural hematoma. JAMA Neurol 2020;77:65-72.

9 Guglielminotti J, Landau R, Li G. Major neurologic complications associated with postdural puncture headache in obstetrics: a retrospective cohort study. Anesth Analg 2019;129:1328-36.

10 Newrick P, Read D. Subdural haematoma as a complication of spinal anaesthetic. BMJ 1982:285:341-2.

11 Amorim JA, Remígio DSCdosA, Damázio Filho 0, et al. Intracranial subdural hematoma post-spinal anesthesia: report of two cases and review of 33 cases in the literature. Rev Bras Anestesiol 2010:60:620-9.

12 Crombie IK. Critical appraisal of a case study. Available: http://www.cebma.org/wpcontent/uploads/Critical-Appraisal-Questions-for-a-Case-Study.pdf [Accessed 1 Aug 2017].

13 Owens WD, Felts JA, Spitznagel EL. ASA physical status classifications: a study of consistency of ratings. Anesthesiology 1978;49:239-43.

14 Teasdale G, Jennett B, Murray L, et al. Glasgow coma scale: to sum or not to sum. Lancet 1983;2:678.

15 Narouze SN, Casanova J, El-Jaberi M, et al. Inadvertent dural puncture during attempted thoracic epidural catheter placement complicated by cerebral and spinal subdural hematoma. J Clin Anesth 2006;18:132-4.

16 Hans GA, Senard M, Ledoux D, et al. Cerebral subarachnoid blood migration consecutive to a lumbar haematoma after spinal anaesthesia. Acta Anaesthesiol Scand 2008;52:1021-3.

17 Rocchi R, Lombardi C, Marradi I, et al. Intracranial and intraspinal hemorrhage following spinal anesthesia. Neurol Sci 2009;30:393-6.

18 Figueroa Arenas MA, Castañeda Rodríguez LY, Pérez Redondo JC, et al. Subdural intracranial and spinal haematoma secondary to neuraxial anaesthesia. Neurologia 2018;33:476-7
19 Edelman JD, Wingard DW. Subdural hematomas after lumbar dural puncture. Anesthesiology 1980;52:167-70.

20 Abstracts of the 36th European Society of Neuroradiology annual meeting. September 21-23, 2012. Edinburgh, Scotland, United Kingdom. Neuroradiology 2012;54(Suppl 1):S33-194

21 Espinosa-Aguilar M, Hernández-Palazón J, Fuentes-García D. Intraspinal and intracranial subarachnoid haemorrhage with severe cerebral vasospasm after spinal anaesthesia for assisted delivery. Br J Anaesth 2012;108:885-6.

22 Mascarinas A, Herman S. Poster 378 rehabilitation of a patient with partial cauda equina syndrome and intracranial hemorrhages following spinal anesthesia for a knee replacement: a case report. $P m$ R 2014;6:S318.

23 Issı Z, Öztürk V, Iyilikçi L, et al. Spinal epidural and intracranial subdural haemorrhage that is a complication of spinal anaesthesia. Turk J Anaesthesiol Reanim 2018;46:319-22.

24 Pitkänen MT, Aromaa U, Cozanitis DA, et al. Serious complications associated with spinal and epidural anaesthesia in Finland from 2000 to 2009. Acta Anaesthesiol Scand 2013:57:553-64.

25 Headache classification Committee of the International headache Society (IHS) the International classification of headache disorders, 3rd edition. Cephalalgia 2018:38:1-211.

26 Davies JM, Murphy A, Smith M, et al. Subdural haematoma after dural puncture headache treated by epidural blood patch. Br J Anaesth 2001;86:720-3.

27 Koc AF, Bozdemir H, Sarica Y, et al. Unilateral subdural hematoma caused by epidural anesthesia. Neurosurg Q 2004;14:41-3.

28 Richa F, Chalhoub V, El-Hage C, et al. Subdural hematoma with cranial nerve palsies after obstetric epidural analgesia. Int J Obstet Anesth 2015;24:390-1.

29 Kale A, Emmez H, Pişkin Özcan, et al. Postdural puncture subdural hematoma or postdural puncture headache?: two cases report. Korean I Anesthesiol 2015;68:509-12.

30 Fujii M, Arai T, Matsuoka Y, et al. [Postpartum chronic subdural hematoma following spinal anesthesia: case report]. No Shinkei Geka 2010;38:563-8.

31 von Knobelsdorff G, Paris A. [Intracerebral hemorrhage after cesarean section under spinal anesthesia. Coincidence or causality?]. Anaesthesist 2004;53:41-4.

32 Wyble SW, Bayhi D, Webre D, et al. Bilateral subdural hematomas after dural puncture: delayed diagnosis after false negative computed tomography scan without contrast. Reg Anesth 1992;17:52-3.

33 Lim G, Zorn JM, Dong YJ, et al. Subdural hematoma associated with labor epidural analgesia: a case series. Reg Anesth Pain Med 2016;41:628-31.

34 Domoto S, Suzuki M, Suzuki S, et al. Subdural hematoma after cesarean delivery without symptoms: a case report. JA Clin Rep 2018;4:18.

35 Rosario LE, Rajan GR. Repeat subdural hematoma after uncomplicated lumbar drain discontinuation: a case report. A\&A Pract 2019;13:107-9.

36 Walega DR, Korn M. Management of a cancer patient with an intrathecal drug delivery system and an acute brain abscess. J Palliat Med 2018;21:727-9.

37 Turnbull DK, Shepherd DB. Post-dural puncture headache: pathogenesis, prevention and treatment. Br J Anaesth 2003:91:718-29.

38 Harris NA. Unrecognised dural punctures--possible mechanisms. Anaesthesia 2008:63:675-6.

39 Corbonnois G, O'Neill T, Brabis-Henner A, et al. Unrecognized dural puncture during epidural analgesia in obstetrics later confirmed by brain imaging. Ann Fr Anesth Reanim 2010;29:584-8.

40 Böttiger BW, Diezel G. [Acute intracranial subarachnoid hemorrhage following repeated spinal anesthesia]. Anaesthesist 1992:41:152-7.

41 Elshanawany AM, Wahab AHA. Intracranial acute subdural hematoma following spinal anesthesia: our experience with six patients. J Neurol Surg A Cent Eur Neurosurg 2020;81:044-7.

42 Bateman BT, Schumacher HC, Bushnell CD, et al. Intracerebral hemorrhage in pregnancy: frequency, risk factors, and outcome. Neurology 2006;67:424-9.

43 Meeks JR, Bambhroliya AB, Alex KM, et al. Association of primary intracerebral hemorrhage with pregnancy and the postpartum period. JAMA Netw Open 2020:3:e202769.

44 Prins M, van Roosmalen J, Scherjon S. Ziekten en afwijkingen die de zwangerschap compliceren. Praktische Verloskunde 2014:279-317.

45 Phillips JMG, Stedeford JC, Hartsilver E, et al. Epidural abscess complicating insertion of epidural catheters. Br J Anaesth 2002;89:778-82. 